\title{
Real-Life Effectiveness and Safety of Sofosbuvir-Based Therapy in Genotype 2 Chronic Hepatitis C Patients in South Korea, with Emphasis on the Ribavirin Dose
}

\author{
Eun Sun Jang ${ }^{1}$, Kyung-Ah Kim², Young Seok Kim ${ }^{3}$, In Hee Kim ${ }^{4}$, Byung Seok Lee ${ }^{5}$, Youn Jae Lee ${ }^{6}$, Woo Jin Chung ${ }^{7}$, and \\ Sook-Hyang Jeong ${ }^{1}$ \\ ${ }^{\prime}$ Department of Internal Medicine, Seoul National University Bundang Hospital, Seoul National University College of Medicine, Seongnam, \\ ${ }^{2}$ Department of Internal Medicine, Inje University Ilsan Paik Hospital, Goyang, ${ }^{3}$ Department of Internal Medicine, Soonchunhyang University \\ Bucheon Hospital, Soonchunhyang University College of Medicine, Bucheon, ${ }^{4}$ Department of Internal Medicine, Chonbuk National University \\ Hospital, Chonbuk National University Medical School, Jeonju, ${ }^{5}$ Department of Internal Medicine, Chungnam National University College of \\ Medicine, Daejeon, ${ }^{6}$ Department of Internal Medicine, Inje University Busan Paik Hospital, Inje University College of Medicine, Busan, and \\ ${ }^{7}$ Department of Internal Medicine, Keimyung University School of Medicine, Daegu, Korea
}

Background/Aims: Sofosbuvir (SOF)-based therapy has been used in Korean patients with chronic hepatitis $C$ virus (HCV) infection since January 2016. This study aimed to investigate the real-life effectiveness and safety of SOF-based therapy in genotype $2 \mathrm{HCV}$ infection. Methods: From January to December 2016, 458 genotype 2 HCV-infected patients who received $\geq 1$ dose of SOF-based therapy were consecutively enrolled in seven tertiary hospitals. Sustained virologic response (SVR) rates and safety were determined by intention-to-treat (ITT) and per-protocol (PP) analyses. Results: The mean age of the patients was 61.0 years; 183 (40\%) were male, and $13.1 \%$ showed a high viral load (>6,000,000 IU/ $\mathrm{mL}$ ). Among the 378 treatment-naïve patients, the SVR rates were $94.2 \%$ (ITT) and $96.7 \%$ (PP). Among the 80 treatmentexperienced patients, the SVR rates were 96.3\% (ITT) and $98.7 \%$ (PP). Patients with a relatively high fibrosis-4 index score $(>3.25)$ had similar SVR rates to those with a relatively low score $(p=0.756)$. A total of 314 patients $(68.6 \%)$ were treated with a reduced ribavirin dose at the prescriber's discretion, but they showed similar SVR rates to those treated with the weight-based dose (ITT: 95.5\% and 92.3\%, PP: $97.4 \%$ and $96.3 \%$, respectively). Adverse events were observed in 191 patients (41.7\%), including 86 (18.8\%) with anemia, but only one (0.2\%) discontinued antiviral therapy due to nausea. Conclusions: SOF-based therapy showed high real-life efficacy and tolerability in Korean patients with genotype 2 chronic HCV infection, regardless of previous antiviral treatment experience and fibrosis score. A reduced ribavirin dose can be considered in this patient cohort. (Gut Liver 2020;14:775-782)

Key Words: Hepatitis C; Chronic; Sofosbuvir; Ribavirin; Genotype 2

\section{INTRODUCTION}

Hepatitis C virus (HCV)-related liver disease is a major health problem, with >185 million infected people and approximately 350,000 HCV-related deaths annually worldwide. ${ }^{1,2}$ In South Korea, the prevalence of anti-HCV is reported to be $0.78 \%$, ${ }^{3}$ and HCV is the second leading cause of liver cirrhosis (LC) and hepatocellular carcinoma. ${ }^{4,5}$

Sofosbuvir (SOF), a nucleotide NS5B polymerase inhibitor, has been used to treat genotype $2 \mathrm{HCV}$ infection in combination with ribavirin (RBV) globally. ${ }^{6}$ Although more potent pangenotypic direct acting antiviral agents including, glecaprevir/pibrentasvir, ${ }^{7,8}$ and SOF/velpatasvir ${ }^{9,10}$ have been developed, SOF and RBV combination therapy was the only regimen for Korean genotype 2 patients until August 2018. In the phase 3 trials, the sustained virologic response (SVR) rates of SOF+RBV therapy for genotype 2 chronic hepatitis patients were reported to be 96\%-100\% for treatment-naïve (TN) $)^{11-13}$ and 90\% for treatmentexperienced (TE) patients. ${ }^{12}$ However, SVR rates in LC patients were reported to be $91 \%-100 \%$ in $\mathrm{TN}^{11,12}$ and $60 \%-78 \%$ in $\mathrm{TE}^{14,15}$ patients.

Moreover, concomitant administration of RBV, which is a

Correspondence to: Sook-Hyang Jeong

Department of Internal Medicine, Seoul National University Bundang Hospital, 82 Gumi-ro 173beon-gil, Bundang-gu, Seongnam 13620, Korea

Tel: +82-31-787-7034, Fax: +82-31-787-4052, E-mail: jsh@snubh.org

Received on July 22, 2019. Revised on September 18, 2019. Accepted on October 26, 2019. Published online February 3, 2020.

pISSN 1976-2283 eISSN 2005-1212 https://doi.org/10.5009/gnl19260

@ This is an Open Access article distributed under the terms of the Creative Commons Attribution Non-Commercial License (http://creativecommons.org/licenses/by-nc/4.0) which permits unrestricted non-commercial use, distribution, and reproduction in any medium, provided the original work is properly cited. 
Table 1. Baseline Characteristics of the Study Population

\begin{tabular}{|c|c|}
\hline Characteristic & Genotype 2 (n=458) \\
\hline Age, yr & $61.0 \pm 11.5$ \\
\hline Male sex & $183(40.0)$ \\
\hline HCV RNA viral load & $6.06 \times 10^{6}\left(15-3.21 \times 10^{7}\right)$ \\
\hline$>6,000,000 \mathrm{IU} / \mathrm{mL}$ & $60(13.1)$ \\
\hline HBV coinfection & $7(1.5)$ \\
\hline \multicolumn{2}{|l|}{ Laboratory findings } \\
\hline $\mathrm{WBC}, / \mathrm{mm}^{3}$ & $5,080(1,074-17,600)$ \\
\hline Hemoglobin, g/dL & $13.6(8.8-17.5)$ \\
\hline PLT, $\times 10^{3} / \mathrm{mm}^{3}$ & $168(33-592)$ \\
\hline $\mathrm{Cr}, \mathrm{mg} / \mathrm{dL}$ & $0.76(0.38-2.10)$ \\
\hline Albumin, $\mathrm{g} / \mathrm{dL}$ & $4.2(1.7-4.9)$ \\
\hline Bilirubin, mg/dL & $0.72(0.1-2.4)$ \\
\hline ALP, IU/L & 89 (26-728) \\
\hline AST, IU/L & $44(6-784)$ \\
\hline ALT, IU/L & $35(6-433)$ \\
\hline GGT, IU/L & $34(2-425)$ \\
\hline PT, INR & $1.06(0.92-1.56)$ \\
\hline \multicolumn{2}{|l|}{ Liver disease status } \\
\hline $\mathrm{CHC}$ & $339(74.0)$ \\
\hline LC, compensated & $101(22.0)$ \\
\hline LC, decompensated & $4(0.9)$ \\
\hline HCC & $11(2.4)$ \\
\hline Liver TPL & $3(0.7)$ \\
\hline \multicolumn{2}{|c|}{ Previous antiviral treatment } \\
\hline Naïve & $378(82.5)$ \\
\hline Experienced & $80(17.5)$ \\
\hline \multicolumn{2}{|l|}{ FIB-4 $(n=435)$} \\
\hline$<1.45$ & 73 (15.9) \\
\hline $1.45-3.25$ & $165(36.0)$ \\
\hline$>3.25$ & $197(43.0)$ \\
\hline \multicolumn{2}{|l|}{ Treatment duration, wk } \\
\hline$<12$ & $18(3.9)$ \\
\hline 12 & 375 (81.9) \\
\hline$>12-16$ & $54(11.8)$ \\
\hline 24 & $11(2.4)$ \\
\hline \multicolumn{2}{|c|}{ Dose of ribavirin (initial), mg/day } \\
\hline$<800$ & $30(6.5)$ \\
\hline 800 & $165(36.0)$ \\
\hline$>800 \&<1,000$ & $1(0.2)$ \\
\hline 1,000 & $236(51.5)$ \\
\hline$>1,000 \&<1,200$ & 0 \\
\hline 1,200 & $26(5.9)$ \\
\hline \multicolumn{2}{|c|}{ Dose of ribavirin (mean), mg/day } \\
\hline$<800$ & $57(12.5)$ \\
\hline 800 & $120(26.3)$ \\
\hline$>800 \&<1,000$ & $56(12.3)$ \\
\hline
\end{tabular}

Table 1. Continued

\begin{tabular}{lc}
\hline \multicolumn{1}{c}{ Characteristic } & Genotype $2(\mathrm{n}=458)$ \\
\hline 1,000 & $199(43.5)$ \\
$>1,000 \&<1,200$ & $9(2.0)$ \\
1,200 & $16(3.5)$ \\
Recommended dose & $143(31.2)$ \\
Weight-based dose* & $314(68.6)$ \\
Treatment completion & \\
Yes & $439(95.9)$ \\
No & $19(4.1)$ \\
Viral breakthrough & $1(0.2)$ \\
Intolerance & $1(0.2)$ \\
Unaffordability & $3(0.7)$ \\
Non-liver related death & $1(0.2)$ \\
Follow-up loss & $13(2.8)$ \\
\hline
\end{tabular}

Data are presented as the mean \pm SD, number (\%), or median (range). $\mathrm{HCV}$, hepatitis C virus; HBV, hepatitis C virus; WBC, white blood cells; PLT, platelet; ALP, alkaline phosphatase; AST, aspartate aminotransferase; ALT; alanine aminotransferase; GGT, gamma-glutamyltransferase; PT, prothrombin time; INR, international normalized ratio; CHC, chronic hepatitis C; LC, liver cirrhosis; HCC, hepatocellular carcinoma; TPL, transplantation recipient; FIB-4, fibrosis-4 index.

*The weight-based dose of ribavirin was defined as $1,000 \mathrm{mg}$ daily in patients with a body weight of $<75 \mathrm{~kg}$ and $1,200 \mathrm{mg}$ daily in patients with a body weight of $\geq 75 \mathrm{~kg}$.

synthetic guanosine analog and a prodrug showing an antiviral activity by interfering with the HCV RNA metabolism, resulted in anemia, itching, or other adverse events. In the pegylated interferon-based era, RBV-induced anemia was reported with a varying incidence of $28 \%$ to $52.2 \%{ }^{16-19}$ in Asian patients. However, the solitary effect of RBV dosing on the development of anemia could not be studied because the concomitantly administered interferon can also cause cytopenia. In the direct acting antiviral agent era, the role of RBV dose on SVR and development of anemia can be clearly investigated, but detailed studies on the RBV dose modification are limited.

Therefore, this study aimed to investigate the effectiveness and safety of SOF-based therapy, and the effect of RBV dose modification for genotype $2 \mathrm{HCV}$ infection in a real-world setting in South Korea.

\section{MATERIALS AND METHODS}

\section{Subjects}

This retrospective study consecutively enrolled 458 patients with genotype $2 \mathrm{HCV}$ infection who received at least one dose of SOF-based therapy from January to December 2016 from seven tertiary hospitals, located in different major regions of South Korea. SOF was administered orally at a dose of $400 \mathrm{mg}$ once daily. RBV was administered orally twice daily, with recommended dose $(1,000 \mathrm{mg}$ daily in patients with a body weight 
of $<75 \mathrm{~kg}$, and $1,200 \mathrm{mg}$ daily in patients with a body weight of $\geq 75 \mathrm{~kg}$ ) according to the label. Nonetheless, the changed dose was accepted in this study with the attending physician's decision during the antiviral treatment, modifying it based on the patients' hemoglobin level and comorbidities.

Patients who had been treated with interferon/peginterferonbased therapy or other direct acting antiviral agents except SOF-based therapy at least one dose were categorized into the TE group. This study was approved by the institutional review board of the seven hospitals, and the requirement for obtaining written informed consent was waived because this study was based on the retrospective review of the existing medical records.

Chronic hepatitis C was defined as the persistence of HCV RNA for more than 6 months regardless of aminotransferase levels. The diagnosis of LC was based on histologic findings or by $\geq 1$ clinical findings of portal hypertension, ${ }^{20}$ which are as follows: (1) cirrhotic appearance of the liver with splenomegaly on imaging study (ultrasonography, computed tomography, or magnetic resonance imaging); (2) low platelet count $(<120,000 /$ $\mathrm{mm}^{3}$ ); (3) presence of varices on endoscopy; (4) presence of ascites; and (5) presence of hepatic encephalopathy. Decompensated LC was defined as the presence of jaundice (total bilirubin $>2$

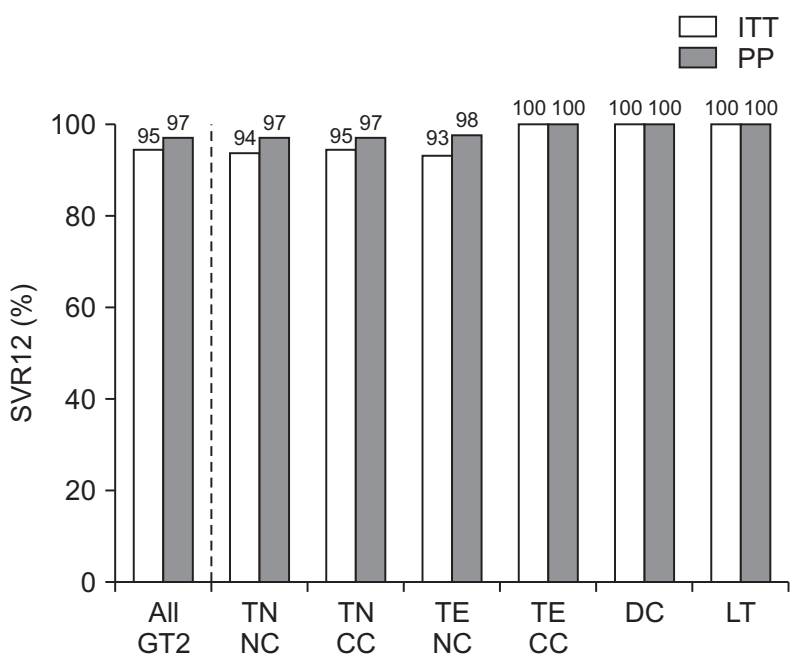

Fig. 1. Sustained virologic response rates of sofosbuvir-based therapy for Korean patients infected with genotype 2 hepatitis $\mathrm{C}$ virus according to their treatment experience and liver disease status.

ITT, intention-to-treat; PP, per-protocol; SVR12, sustained virologic response at 12 weeks after end of treatment; GT2, genotype 2; TN NC, treatment-naïve patients with no cirrhosis; TN CC, treatment-naïve patients with compensated cirrhosis; TE NC, treatment-experienced patients with no cirrhosis; TE CC, treatment-experienced patients with compensated cirrhosis; DC, patients with decompensated cirrhosis; LT, liver transplantation recipients.

Table 2. Treatment Duration of Sofosbuvir Plus Ribavirin Therapy According to Underlying Liver Disease and Treatment Experience

\begin{tabular}{|c|c|c|c|c|c|}
\hline \multirow{2}{*}{ Regimen } & \multicolumn{4}{|c|}{ Duration, wk } & \multirow{2}{*}{ Subtotal } \\
\hline & $<12$ & 12 & $>12-16$ & 24 & \\
\hline \multicolumn{6}{|l|}{$\mathrm{SOF}+\mathrm{RBV}$} \\
\hline TN NC & $14(4.8)$ & $269(92.8)$ & $5(1.7)$ & $2(0.7)$ & $290(65.0)$ \\
\hline TN CC & $2(2.8)$ & 39 (54.9) & 24 (33.8) & $6(8.5)$ & 71 (15.9) \\
\hline TE NC & $2(4.7)$ & 40 (93.0) & $1(2.3)$ & - & $43(9.6)$ \\
\hline TE CC & - & $11(31.4)$ & $21(60.0)$ & $3(8.6)$ & $35(7.8)$ \\
\hline $\mathrm{DC}$ & - & $1(25.0)$ & $3(75.0)$ & - & $4(0.9)$ \\
\hline LT & - & $3(100)$ & - & - & $3(0.7)$ \\
\hline Subtotal & $18(4.1)$ & $363(81.4)$ & 54 (12.1) & $11(2.5)$ & 446 (100) \\
\hline \multicolumn{6}{|l|}{$\mathrm{SOF}+\mathrm{DCV}$} \\
\hline TN CC & - & $1(100)$ & - & - & $1(100)$ \\
\hline Subtotal & - & $1(100)$ & - & - & $1(100)$ \\
\hline \multicolumn{6}{|c|}{$\mathrm{SOF}+\mathrm{DCV}+\mathrm{RBV}$} \\
\hline TN NC & - & $2(100)$ & - & - & $2(50)$ \\
\hline TN CC & - & $2(100)$ & - & - & $2(50)$ \\
\hline Subtotal & - & $4(100)$ & - & - & $4(100)$ \\
\hline \multicolumn{6}{|l|}{ SOF } \\
\hline TN NC & - & $6(100)$ & - & - & $6(85.7)$ \\
\hline TN CC & - & $1(100)$ & - & - & $1(14.3)$ \\
\hline Subtotal & - & $7(100)$ & - & - & $7(100)$ \\
\hline
\end{tabular}

Data are presented as number (\%).

SOF, sofosbuvir; RBV, ribavirin; TN NC, treatment-naïve patients with no cirrhosis; TN CC, treatment-naïve patients with compensated cirrhosis; TE NC, treatment-experienced patients with no cirrhosis; TE CC, treatment-experienced patients with compensated cirrhosis; DC, patients with decompensated liver cirrhosis; LT, liver transplantation recipients; DCV, daclatasvir. 
$\mathrm{mg} / \mathrm{dL}$ ), ascites (including controlled ascites by diuretics), and previous history of variceal bleeding or portosystemic encephalopathy. Additionally, fibrosis-4 index (FIB-4) was calculated according to the developed equation ${ }^{21}$ as an indirect marker of hepatic fibrosis.

\section{Measurements for treatment response and adverse events of antiviral therapy}

HCV RNA concentration was measured by a real-time polymerase chain reaction assay at the laboratory medicine department of each study site. Treatment responses were documented according to the clinical practice guideline for hepatitis $C$ by the Korean Association for the Study of the Liver. ${ }^{6}$ SVR was defined as undetectable HCV RNA at 12 weeks after completion of treatment. Viral breakthrough referred to the reappearance of
HCV RNA during the treatment, and relapse was defined as the reappearance of HCV RNA after initial viral elimination with undetectable HCV RNA level at the end of treatment. In patients who could not be tested for HCV RNA at the exact weeks for SVR measurement, the window period of \pm 4 weeks was permitted.

The adverse events were based on chart review by the attending physician. Owing to the limitation of the study's retrospective design, the grade of adverse events could not be evaluated.

\section{Statistical analysis}

Analyses were conducted by descriptive statistics for virological outcomes and safety. SVR with intention-to-treat (ITT) analysis was calculated for all enrolled patients $(n=458)$ and with per-protocol (PP) analysis for subjects who completed
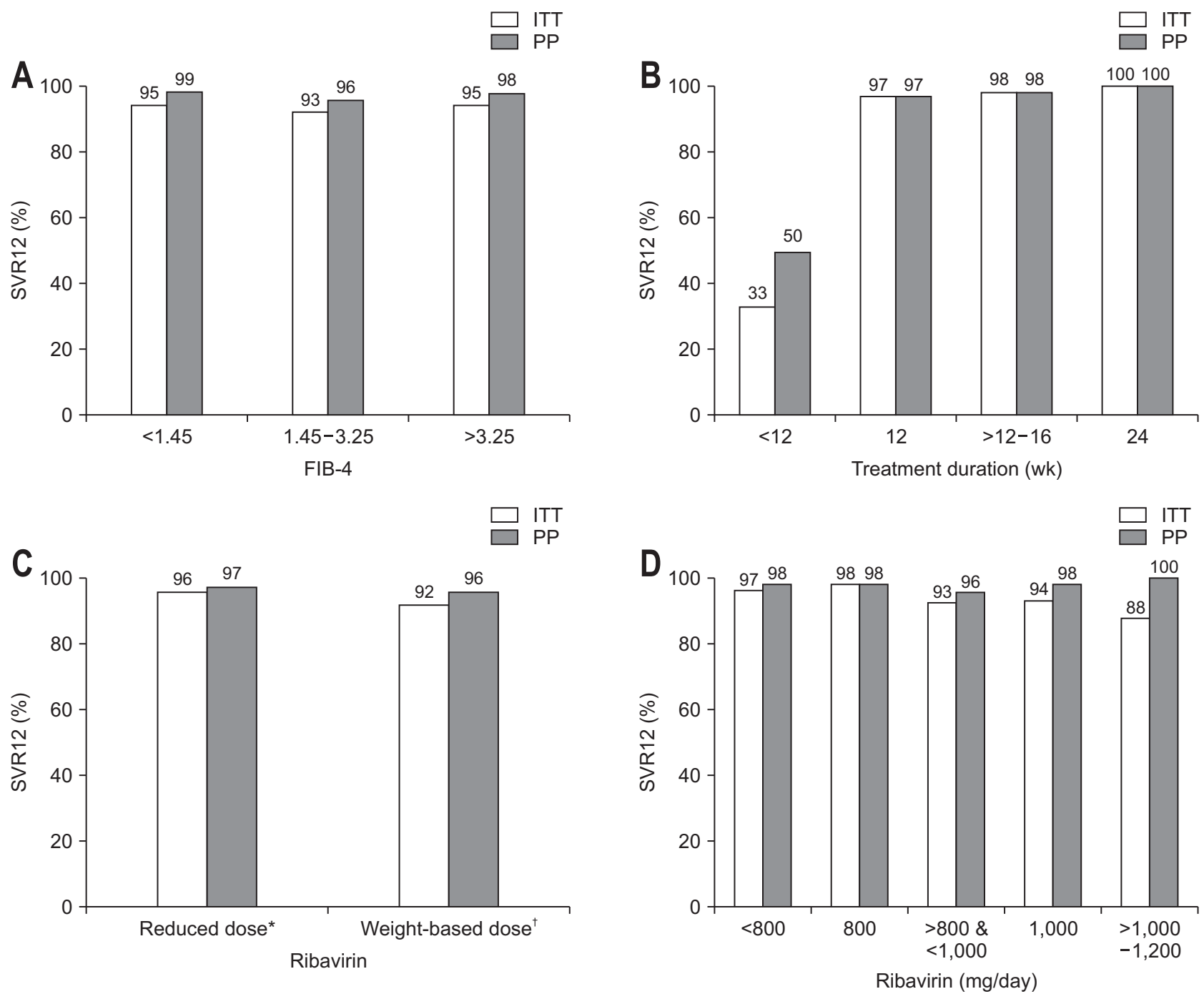

Fig. 2. Sustained virologic response rates of sofosbuvir-based therapy for Korean patients infected with genotype 2 hepatitis $C$ virus according to the FIB-4 (A), treatment duration (B), and dose of ribavirin (C, D).

ITT, intention-to-treat; PP, per-protocol; SVR12, sustained virologic response at 12 weeks after end of treatment; FIB-4, fibrosis-4 index. *The lower dosage of ribavirin than the weight-based recommendation was defined as the reduced dose; ${ }^{\dagger}$ The weight-based dose of ribavirin was defined as $1,000 \mathrm{mg}$ daily in patients with a body weight of $<75 \mathrm{~kg}$ and $1,200 \mathrm{mg}$ daily in patients with a body weight of $\geq 75 \mathrm{~kg}$. 
the scheduled treatment and follow-up for $\geq 12$ weeks after the treatment completion $(n=440)$. Patients who had withdrawn antiviral treatment according to the attending physician's decision owing to the inadequate virologic response or significant adverse events were included in the PP analysis, regardless of the follow-up duration after the cessation of treatment. All statistical analyses were performed using Stata version 14.0 (College Station, TX, USA).

\section{RESULTS}

\section{Baseline patient characteristics}

Among the 458 participants, mean age was 61.0 years, 40\% were male, and 23\% had LC (22.1\% compensated and 0.9\% decompensated). The four decompensated LC patients were categorized into this group because their Child-Pugh class were $\mathrm{B}$ owing to the presence of ascites but it was well controlled with diuretics. A total of 378 (82.5\%) were naïve for antiviral treatment, and $80(17.5 \%)$ had experience with other antiviral therapy before the SOF-based treatment (Table 1).

With SOF+RBV regimen, 92.8\% of TN and 93\% of TE noncirrhotic (NC) patients were treated for 12 weeks (Table 2). Among the compensated cirrhotic (CC) patients, 42.3\% of TN and 68.6\% of TE patients were treated for more than 12 weeks (Table 2). Twelve patients were treated with therapies other than the SOF+RBV regimen (1 SOF+daclatasvir, 4 SOF+daclatasvir+RBV, and 7 SOF only).

\section{SVR rates of SOF-based therapy}

Among the 458 patients (ITT population), 18 (3.9\%) were lost to follow-up during or after the scheduled treatment and could not be evaluated for SVR (440 PP population). Overall, the SVR rates of Korean genotype 2 patients treated with SOF-based regimen were $94.5 \%(433 / 458)$ in ITT and 97.1\% (427/440) in PP analysis (Fig. 1). Among the 13 patients who did not achieve SVR, four were not tested for SVR within 16 weeks after treatment completion, and clinical characteristics of the remaining nine patients are listed in Supplementary Table 1.

TN patients showed SVR rates of 94.2\% (356/378) in ITT and 96.7\% (350/362) in PP analyses, and TE patients showed 96.3\% (77/80) in ITT and 98.7\% (77/78) in PP analyses (data not shown). NC patients showed SVR rates of 93.8\% (318/339) in ITT and 96.6\% (313/324) in PP analyses, and cirrhotic patients without decompensation (CC) showed 97.0\% (98/101) in ITT and 99.0\% (97/98) in PP analyses (data not shown).

Among TN patients, NC and CC did not show a significant difference in the SVR rates $(94.0 \%$ vs $94.5 \%$ in ITT, $p=0.609$; $96.5 \%$ vs $97.1 \%$ in PP, $\mathrm{p}=0.606$ ) (Fig. 1, Supplementary Table 2). Among TE patients, the existence of compensated cirrhosis did not affect the SVR rates (NC vs CC: $93.3 \%$ vs 100\% in ITT, $\mathrm{p}=0.364 ; 97.7 \%$ vs $100 \%$ in PP, $\mathrm{p}=0.364$ ) (Fig. 1, Supplementary Table 2). Four decompensated cirrhotic patients and three liver transplant recipients had SVR of 100\% (Fig. 1, Supplementary Table 2). Among the four decompensated cirrhotic patients, three tolerated 1,000 mg/day of RBV and one had reduced RBV dose of $600 \mathrm{mg} /$ day.

Among the 12 patients who were treated with regimens other than SOF+RBV, 11 achieved SVR at 12 weeks (SVR12) (1 TN CC with SOF+DCV, 2 TN NC and 2 TN CC with SOF+DCV+RBV, 5 TN NC and 1 TN CC with SOF monotherapy), whereas one 70-year-old TN NC patient with FIB-4 of 2.03 treated with 12 weeks of SOF monotherapy failed to achieve SVR12 (data not shown).

\section{Effect of FIB-4, treatment duration, and dose of RBV on the SVR rates of SOF-based therapy}

As shown in Fig. 2A, FIB-4 did not significantly affect the SVR rate in Korean genotype 2 patients treated with the SOFbased regimen $(\mathrm{p}=0.756)$ (Supplementary Table 2). Each group based on the FIB-4 score $^{21}(<1.45,1.45-3.25$, and $>3.25)$ showed favorable SVR even with advanced fibrosis. Fig. 2B showed that the short duration of SOF-based therapy $<12$ weeks (shortened therapy or early withdrawal with various reasons before 12 weeks) showed a poor SVR rate of $<50 \%$. Although most patients who were treated longer than 12 weeks had cirrhosis, they achieved a high SVR rate with prolonged therapy, similar

Table 3. All Grades of Adverse Events during Sofosbuvir-Based Therapy in Genotype 2 Hepatitis C Patients

\begin{tabular}{lc}
\hline \multicolumn{1}{c}{ Variable } & No. (\%) \\
\hline Any grade of adverse events & $191(41.7)$ \\
Anemia & $86(18.8)$ \\
Hemoglobin $<10.0 \mathrm{~g} / \mathrm{dL}$ & $86(18.8)$ \\
Hemoglobin $<8.5 \mathrm{~g} / \mathrm{dL}$ & $18(3.9)$ \\
Fatigue \& general weakness & $41(9.0)$ \\
Headache & $32(7.0)$ \\
Dyspepsia & $31(6.8)$ \\
Itching & $29(6.3)$ \\
Dizziness & $29(6.3)$ \\
Nausea & $22(4.8)$ \\
Flu-like symptoms & $18(3.9)$ \\
Rash & $16(3.5)$ \\
Dyspnea & $15(3.3)$ \\
Insomnia & $9(2.0)$ \\
Vomiting & $7(1.5)$ \\
Diarrhea & $6(1.3)$ \\
Epigastric soreness & $6(1.3)$ \\
Cough & $5(1.1)$ \\
Anorexia & $5(1.1)$ \\
Depression & $2(0.4)$ \\
Alopecia & $2(0.4)$ \\
Hematuria & $2(0.4)$ \\
\hline
\end{tabular}


to NC patients treated for 12 weeks (Fig. 2B, Supplementary Table 2). Notably, 196 of the patients (42.8\%) started antiviral therapy with RBV of $<1,000 \mathrm{mg} /$ day despite their normal hemoglobin level based on the physician's preference. The proportion of patients requiring RBV dose reduction during treatment was $68.6 \%$ (Table 1). Nonetheless, patients treated with the reduced dose of RBV showed similar SVR rates to those treated with the recommended dose of RBV (Fig. 2C and D, Supplementary Table 2).

\section{Treatment withdrawal and adverse events during SOF- based therapy in Korean HCV-infected patients}

A total of 19 patients discontinued treatment before the completion of the scheduled SOF-based therapy. Virologic failure was found in one patient who showed viral breakthrough at 8 weeks, the remainder discontinued therapy due to adverse events. Three subjects withdrew the treatment due to cost, and one died with pneumonia. An additional 13 were lost to followup during the treatment (Table 1).

Any grade of adverse events were found in 41.7\% of the participants (Table 3). The most common symptoms were anemia (18.8\%), fatigue/general weakness (9.0\%), headache (7.0\%), dyspepsia (6.8\%), and itching (6.3\%). No adverse event accounted for $>10 \%$, except for anemia, and most were tolerable with supportive management. Only one patient discontinued the therapy due to nausea and dyspepsia.

\section{DISCUSSION}

In this retrospective real-life study of SOF-based therapy for genotype $2 \mathrm{HCV}$-infected Korean patients, the SVR rates were high (94.5\% in ITT and 97.1\% in PP) and the underlying liver disease severity and previous treatment experience did not significantly affect the SVR rates. Likewise, patients with a high FIB-4 score showed comparable SVR rates with those with a low FIB-4 score. Notably, patients with very short treatment duration ( $<12$ weeks) or without RBV showed lower SVR rates. However, SVR rates in those treated with $<1,000 \mathrm{mg} /$ day of RBV combined with SOF did not differ from those treated with the recommended dose.

According to a previous real-life study using the HCVTARGET database, ${ }^{22}$ SVR for genotype 2 patients undergoing SOF+RBV therapy was $72 \%$, which is much lower than our result. Additionally, treatment completion rate in the HCVTARGET was lower (90.6\%) than our data (95.9\%). In another study with a high treatment completion rate, ${ }^{23-25}$ the real-life SVR with SOF+RBV in genotype 2 patients was 95\% to 98\% in TN patients, 91.2\% in TE non-responders, and 100\% in TE relapse patients, which were comparable with the findings in the present study.

Liver disease severity is an important factor for successful SVR with antiviral therapy in HCV-infected patients; thus, as- sessment of the fibrosis stage before the selection of treatment regimen and duration is recommended. ${ }^{26}$ A recent Japanese multicenter cohort study revealed an SVR rate of 90.8\% in patients with high FIB-4, which was significantly lower compared to $98.1 \%$ of those with low FIB- 4 after SOF+RBV therapy. ${ }^{27}$ The reason for the universally high SVR rate in NC (97.6\%) or cirrhotic (99.1\%) Korean patients might be the longer treatment duration for cirrhosis compared to that of Japanese patients who were only allowed 12 weeks of treatment due to reimbursement limitations for SOF-based treatment in Japan. Moreover, four decompensated cirrhotic patients showed SVR rate of 100\% without any significant adverse event. Additionally, the fibrosis stage based on FIB-4 score did not significantly affect the SVR rate (Fig. 2A). These findings were correlated with high completion rate of patients with advanced cirrhosis. Treatment withdrawal rates were $4.7 \%$ in NC and $2.7 \%$ in cirrhotic patients, and 5.5\%, 6.1\%, and 2.5\% in patients with FIB-4 score of $<1.45$, 1.45-3.25, and $>3.25$, respectively (data not shown). This result suggested that the adherence to the antiviral treatment, which was not considered in clinical studies, is more crucial than the liver disease severity for achieving successful antiviral eradication in real-life settings.

Detailed prescription pattern of RBV was not investigated in previous studies because practice guidelines ${ }^{6,26}$ recommended two fixed doses (1,000 or 1,200 mg/day) based on body weight. Nonetheless, our data showed that more than $1 / 3$ of the physicians started RBV with a dose of $800 \mathrm{mg} /$ day (Table 1), which was likely based on their experience in treating with pegylated interferon. ${ }^{28}$ Moreover, the weight-based dosing had to be reduced during the whole duration of the treatment in $68.6 \%$ (Table 1). Reduced dosing of RBV was more frequent in patients weighing $<75 \mathrm{~kg}$ (45.4\% vs 7.6\%, p $<0.001$, data not shown) and those with chronic kidney disease grade 3 or more $(90.5 \%$ [19/21] vs 68.2\% [276/403], p=0.031, data not shown). In Asian patients, the RBV-induced anemia was more frequently reported $(37.0 \%)$ in a previous study related to inosine triphosphatase polymorphism, ${ }^{29}$ compared to Caucasian (25.8\%) or Hispanic (20.4\%) patients. Thus, the RBV dose reduction rate was high (35.6\%) in the setting of peginterferon and RBV combination therapy. ${ }^{30}$ Nonetheless, our data showed the SVR rate in Korean patients receiving the reduced dose of RBV (Fig. 2C) was not inferior to that in patients receiving the full weight-based dose. Moreover, the efficacies of stratified RBV dosing were not significantly different between those with weighted $<75$ and those $\geq 75 \mathrm{~kg}$ (Supplementary Fig. 1). Thus, the relevant dose reduction of RBV resulted in better treatment compliance and subsequent successful viral eradication.

There are several limitations of this study. First, the reason for treatment withdrawal was not fully documented because most patients did not visit the study site after the final prescription; thus, the possible problem that caused treatment intolerance might be underestimated. Secondly, the frequency of adverse 
events was based on medical records and might be incompletely categorized owing to the different tolerability of the patients. However, the most recorded symptom did not result in treatment discontinuation. Thirdly, the reason of RBV dose selection was not investigated owing to the study's retrospective design. However, the lower RBV dose was related to older age (i.e., mean of $68.7 \pm 10.5$ years in patients with RBV dose of $<800 \mathrm{mg} /$ day and $53.7 \pm 8.3$ years in patients with RBV dose of $1,200 \mathrm{mg} /$ day, $\mathrm{p}<0.0001$, data not shown), lower body weight $(p=0.0009)$, lower baseline hemoglobin level $(p<0.001)$ and platelet count $(\mathrm{p}=0.004)$, and more frequent LC $(\mathrm{p}=0.005$, data not shown), which are well-known predictive factors of RBVinduced anemia. ${ }^{31}$ This implies that the dose of RBV was based on the patient's comorbidity and expected tolerance as decided by an experienced physician.

In conclusion, SOF-based therapy showed a high real-life efficacy and tolerable safety in Korean patients with genotype 2 chronic HCV infection, regardless of previous antiviral treatment experience and fibrosis score. A reduced dose of RBV combined with SOF is as effective as its standard dose in genotype 2 Asian patients.

\section{CONFLICTS OF INTEREST}

No potential conflict of interest relevant to this article was reported.

\section{ACKNOWLEDGEMENTS}

This study was supported by a grant of Gilead (IN-US-334-4003), and a grant for the Chronic Infectious Disease Cohort Study (Korea HCV Cohort Study, 4800-4859-304) from the Korea Centers for Disease Control and Prevention.

The authors are grateful to the devoted research coordinators (Da-Seul Lee, Dawoon Jeong, and Seon Young Park from Seoul National University Hospital, Seongnam; Hyoyoung Kang from Chonbuk National University Hospital, Chonju; Semi Jeon from Soonchunhyang University Bucheon Hospital, Bucheon; Dalli Nam from Inje University Busan Paik Hospital, Busan; Su Jin Lee from Keimyung University Dongsan Medical Center, Daegu; and Young Soon Lee from Inje University Ilsan Paik Hospital, Goyang).

\section{AUTHOR CONTRIBUTIONS}

Study concept and design: E.S.J., S.H.J. Data acquisition: E.S.J., K.A.K., Y.S.K., I.H.K., B.S.L., Y.J.L., W.J.C., S.H.J. Data analysis and interpretation: E.S.J., S.H.J. Drafting of the manuscript: E.S.J. Critical revision of the manuscript for important intellectual content: K.A.K., Y.S.K., I.H.K., B.S.L., Y.J.L., W.J.C. Statistical analysis: E.S.J. Obtained funding and study supervision: S.H.J.

\section{ORCID}

Eun Sun Jang

Kyung-Ah Kim

Young Seok Kim

In Hee Kim

Byung Seok Lee

Youn Jae Lee

Woo Jin Chung

Sook-Hyang Jeong

https://orcid.org/0000-0003-4274-2582

https://orcid.org/0000-0002-6128-6407

https://orcid.org/0000-0002-7113-3623

https://orcid.org/0000-0003-3863-7907

https://orcid.org/0000-0002-4669-8892

https://orcid.org/0000-0003-3854-3388

https://orcid.org/0000-0002-3736-1067

https://orcid.org/0000-0002-4916-7990

\section{REFERENCES}

1. Mohd Hanafiah K, Groeger J, Flaxman AD, Wiersma ST. Global epidemiology of hepatitis C virus infection: new estimates of age-specific antibody to HCV seroprevalence. Hepatology 2013;57:1333-1342.

2. Stanaway JD, Flaxman AD, Naghavi M, et al. The global burden of viral hepatitis from 1990 to 2013: findings from the Global Burden of Disease Study 2013. Lancet 2016;388:1081-1088.

3. Kim DY, Kim IH, Jeong SH, et al. A nationwide seroepidemiology of hepatitis C virus infection in South Korea. Liver Int 2013;33:586-594.

4. Lee SS, Byoun YS, Jeong SH, et al. Type and cause of liver disease in Korea: single-center experience, 2005-2010. Clin Mol Hepatol 2012;18:309-315.

5. Seong MH, Kil H, Kim YS, et al. Clinical and epidemiological features of hepatitis C virus infection in South Korea: a prospective, multicenter cohort study. J Med Virol 2013;85:1724-1733.

6. Korean Association for the Study of the Liver (KASL). 2017 KASL clinical practice guidelines management of hepatitis $\mathrm{C}$ : treatment of chronic hepatitis C. Clin Mol Hepatol 2018;24:169-229.

7. Toyoda H, Chayama K, Suzuki F, et al. Efficacy and safety of glecaprevir/pibrentasvir in Japanese patients with chronic genotype 2 hepatitis C virus infection. Hepatology 2018;67:505-513.

8. Asselah T, Kowdley KV, Zadeikis N, et al. Efficacy of glecaprevir/ pibrentasvir for 8 or 12 weeks in patients with hepatitis $\mathrm{C}$ virus genotype 2, 4, 5, or 6 infection without cirrhosis. Clin Gastroenterol Hepatol 2018;16:417-426.

9. Jacobson IM, Lawitz E, Gane EJ, et al. Efficacy of 8 weeks of sofosbuvir, velpatasvir, and voxilaprevir in patients with chronic HCV infection: 2 phase 3 randomized trials. Gastroenterology 2017;153:113-122.

10. Bourlière M, Gordon SC, Flamm SL, et al. Sofosbuvir, velpatasvir, and voxilaprevir for previously treated HCV infection. N Engl J Med 2017;376:2134-2146.

11. Lawitz E, Mangia A, Wyles D, et al. Sofosbuvir for previously untreated chronic hepatitis C infection. N Engl J Med 2013;368:18781887.

12. Ahn SH, Lim YS, Lee KS, et al. A phase 3b study of sofosbuvir plus ribavirin in treatment-naive and treatment-experienced Korean patients chronically infected with genotype 2 hepatitis $C$ virus. 
J Viral Hepat 2016;23:358-365.

13. Omata M, Nishiguchi S, Ueno Y, et al. Sofosbuvir plus ribavirin in Japanese patients with chronic genotype $2 \mathrm{HCV}$ infection: an open-label, phase 3 trial. J Viral Hepat 2014;21:762-768.

14. Jacobson IM, Gordon SC, Kowdley KV, et al. Sofosbuvir for hepatitis C genotype 2 or 3 in patients without treatment options. $\mathrm{N}$ Engl J Med 2013;368:1867-1877.

15. Zeuzem S, Dusheiko GM, Salupere R, et al. Sofosbuvir and ribavirin in HCV genotypes 2 and 3. N Engl J Med 2014;370:19932001.

16. Kim JS, Ahn SM, Jung YK, et al. The impact of inosine triphosphatase variants on hemoglobin level and sustained virologic response of chronic hepatitis C in Korean. J Korean Med Sci 2013;28:1213-1219.

17. Kurosaki M, Tanaka Y, Tanaka K, et al. Relationship between polymorphisms of the inosine triphosphatase gene and anaemia or outcome after treatment with pegylated interferon and ribavirin. Antivir Ther 2011;16:685-694.

18. Kwon JH, Bae SH, Choi JY, et al. Assessment of the efficacy of reducing peginterferon alpha-2a and ribavirin dose on virologic response in Koreans with chronic hepatitis C. Korean J Intern Med 2009;24:203-211.

19. Lee H, Choi MS, Paik SW, et al. Peginterferon alfa-2a plus ribavirin for initial treatment of chronic hepatitis C in Korea. Korean J Hepatol 2006;12:31-40.

20. Suk KT, Baik SK, Yoon JH, et al. Revision and update on clinical practice guideline for liver cirrhosis. Korean J Hepatol 2012;18:121.

21. Sterling RK, Lissen E, Clumeck N, et al. Development of a simple noninvasive index to predict significant fibrosis in patients with HIV/HCV coinfection. Hepatology 2006;43:1317-1325.

22. Reddy KR, Lim JK, Kuo A, et al. All-oral direct-acting antiviral therapy in HCV-advanced liver disease is effective in real-world practice: observations through HCV-TARGET database. Aliment Pharmacol Ther 2017;45:115-126.

23. Akahane T, Kurosaki M, Itakura J, et al. Real-world efficacy and safety of sofosbuvir + ribavirin for hepatitis C genotype 2: a nationwide multicenter study by the Japanese Red Cross Liver Study Group. Hepatol Res 2019;49:264-270.

24. Kim YM, Kim SB, Song IH, et al. Efficacy and safety of sofosbuvir plus ribavirin for Korean patients with hepatitis $\mathrm{C}$ virus genotype 2 infection: a retrospective multi-institutional study. Clin Mol Hepatol 2018;24:311-318.

25. Ogawa E, Furusyo N, Nomura H, et al. Effectiveness and safety of sofosbuvir plus ribavirin for HCV genotype 2 patients 65 and over with or without cirrhosis. Antiviral Res 2016;136:37-44.

26. European Association for the Study of the Liver. EASL recommendations on treatment of hepatitis C 2018. J Hepatol 2018;69:461511.

27. Morio K, Imamura M, Kawakami Y, et al. Advanced liver fibrosis effects on the response to sofosbuvir-based antiviral therapies for chronic hepatitis C. J Med Virol 2018;90:1834-1840.

28. Korean Association for the Study of the Liver. KASL clinical practice guidelines: management of hepatitis C. Clin Mol Hepatol 2016;22:76-139.

29. Kozuka R, Hai H, Teranishi Y, et al. Correlation between polymorphism in the inosine triphosphatase and the reductions in hemoglobin concentration and ribavirin dose during sofosbuvir and ribavirin therapy. J Gastroenterol Hepatol 2017;32:1495-1502.

30. Hu KQ, Freilich B, Brown RS, Brass C, Jacobson IM. Impact of Hispanic or Asian ethnicity on the treatment outcomes of chronic hepatitis C: results from the WIN-R trial. J Clin Gastroenterol 2011;45:720-726.

31. Borroni G, Cazzaniga M, Andreoletti M, et al. Low glomerular filtration rate is a risk factor for ribavirin-associated anaemia in old patients with chronic hepatitis C. J Viral Hepat 2013;20:e90-e95. 